\title{
On structural, optical and dielectric properties of zinc aluminate nanoparticles
}

\author{
E MUHAMMAD ABDUL JAMAL, D SAKTHI KUMAR ${ }^{\dagger}$ and M R ANANTHARAMAN* \\ Department of Physics, Cochin University of Science and Technology, Cochin 682 022, India \\ ${ }^{\dagger}$ Bio Nano Electronics Research Centre, Toyo University, Tokyo, Japan
}

MS received 22 December 2008

\begin{abstract}
Zinc aluminate nanoparticles with average particle size of $40 \mathrm{~nm}$ were synthesized using a sol-gel combustion method. X-ray diffractometry result was analysed by Rietveld refinement method to establish the phase purity of the material. Different stages of phase formation of the material during the synthesis were investigated using differential scanning calorimetry and differential thermogravimetric analysis. Particle size was determined with transmission electron microscopy and the optical bandgap of the nanoparticles was determined by absorption spectroscopy in the ultraviolet-visible range. Dielectric permittivity and a.c. conductivity of the material were measured for frequencies from $100 \mathrm{kHz}$ to $8 \mathrm{MHz}$ in the temperature range of $30-120^{\circ} \mathrm{C}$. The presence of MaxwellWagner type interfacial polarization was found to exist in the material and hopping of electron by means of quantum mechanical tunneling is attributed as the reason for the observed a.c. conductivity.
\end{abstract}

Keywords. Nanoparticles; dielectric permittivity; interfacial polarization; activation energy.

\section{Introduction}

Zinc aluminate, a mixed oxide of aluminium and zinc, is a naturally available mineral commonly called as gahnite with a normal spinel structure having all zinc cations in the tetrahedral and all aluminium cations in the octahedral voids of $f c c$ lattice of oxygen anions. Zinc aluminate is a widely used catalyst employed in chemical reactions viz. synthesis of methanol and synthesis of styrenes from acetophenones (van der Laag et al 2004; Wei and Chen 2006). In addition to this, zinc aluminate is used as a catalyst support owing to its high thermal stability. Recently the optical properties of zinc aluminate is being investigated and it was reported that polycrystalline zinc aluminate has an optical bandgap nearly equal to $320 \mathrm{~nm}$ and is highly reflective at wavelengths less than $300 \mathrm{~nm}$ (Pandey et al 1999; Ciupina et al 2004). Thus zinc aluminate may find applications in optoelectronic devices operated in the ultraviolet region. Studies on the optical and catalytic properties of zinc aluminate nanoparticles were carried out by many researchers, but reports on the dielectric properties of this material is very rarely found in literature. Zinc aluminates resemble zinc ferrite structurally, and it has been found recently that zinc ferrite in the nano-regime show anomalous magnetic properties in that zinc ions instead of occupying tetrahedral (A) sites occupies octahedral (B) sites partially when prepared in the nano-regime (Roy et al 2006). Zinc aluminate can be considered as the non-magnetic counterpart of zinc ferrite and

\footnotetext{
*Author for correspondence (mraiyer@yahoo.com)
}

an ideal template to test the hypothesis on the migration of zinc ions to the B sites as a consequence of the size reduction as the standard property. This article describes the synthesis of zinc aluminate nanoparticles in a sol-gel combustion method, the characterization of the material and the evaluation of its dielectric properties in a frequency range from $100 \mathrm{kHz}$ to $8 \mathrm{MHz}$. Synthesis of zinc aluminate nanoparticles in sol-gel combustion method was taken as an example for the synthesis of spinel structured mixed oxide nanoparticles in general and it was attempted to understand the various chemical and physical phenomena involved in the process of synthesis with analysis carried out in each stage of the preparation by various analytical tools.

\section{Experimental}

Zinc nitrate hydrate and aluminium nitrate hydrate taken in 2:1 molar ratio were dissolved in ethylene glycol, by adding ethylene glycol in small quantities, to form a saturated solution. The solution was heated at $70^{\circ} \mathrm{C}$ with constant stirring on a magnetic stirrer for several hours until gel formation had taken place following a chemical reaction. The gel was heated at a temperature of $180^{\circ} \mathrm{C}$ placed on a heating mantle until combustion had taken place forming slightly rosecoloured fine powder. This powder was analysed using Xray diffractometry and it was found that the phase of zinc aluminate was not yet formed. This powder was calcinated at different temperatures starting from $350^{\circ} \mathrm{C}$ and had been analysed using XRD after each calcination trials to study the 
structural details. Calcination at $700^{\circ} \mathrm{C}$ for $6 \mathrm{~h}$ yielded phase pure zinc aluminate nanoparticles.

The gel formed at $70^{\circ} \mathrm{C}$ had been analysed by means of differential scanning calorimetry (DSC) (Mettler Toledo DSC 822e) and thermogravimetric analysis (Perkin Elmer, Diamond TG). Zinc aluminate nanocrystallites thus formed were characterized using X-ray diffractometry (XRD) (Rigaku Dmax C with $\mathrm{Cu} \mathrm{K} \alpha \mathrm{X}$-ray source), transmission electron microscopy and energy dispersive spectra (EDS) (Jeol JEM-2200 FS electron microscope operated at $200 \mathrm{kV}$ ), UV-Vis NIR spectroscopy (Jasco V 530 spectrophotometer) and Fourier transform infrared spectroscopy (FTIR) (Thermo Nicolet Avatar 370).

Dielectric and a.c. conductivity studies of zinc aluminate were carried out using HP impedance analyser model $4285 \mathrm{~A}$ and a home-made dielectric cell under a vacuum of $10^{-3}$ mbar and at temperatures varying from $30-120^{\circ} \mathrm{C}$. The fabrication details of the dielectric cell are cited elsewhere (Mohammed and Anantharaman 2002). Nanoparticles of zinc aluminate were pressed into pellets of size $12 \mathrm{~mm}$ in diameter and $\sim 2 \mathrm{~mm}$ in thickness. These pellets were sintered at a temperature of $700^{\circ} \mathrm{C}$ for $24 \mathrm{~h}$ to achieve maximum compaction of the particles. In the dielectric cell a capacitor was formed by keeping the pellet in between two copper discs of the same diameter and the capacitance was measured in the impedance analyser. Temperature was measured with a Cryocon temperature controller with platinum resistance (PT-100) sensor. The measurements of the capacitance of the capacitor were carried out in a frequency range of $100 \mathrm{kHz}-$ $8 \mathrm{MHz}$. The instrument we used is capable of capacitance measurements up to $30 \mathrm{MHz}$, but we had limited the measurements only to $8 \mathrm{MHz}$, because of the limitations of the co-axial cable used in our experiments. The dielectric permittivity of the material was calculated from the values of capacitance using the relation

$$
\varepsilon^{\prime}=\frac{C d}{\varepsilon_{0} A},
$$

and dielectric loss of the material using the equation

$$
\varepsilon^{\prime \prime}=\varepsilon^{\prime} \tan \delta,
$$

where $\varepsilon^{\prime}$ is the dielectric permittivity of the sample (the real part), $C$ the capacitance of the capacitor formed by inserting the sample between two metal plates, $d$ the thickness of the sample, $\varepsilon_{0}$ the permittivity of free space and $A$ the area of cross section of the sample. The a.c. conductivity of the sample was determined from the loss tangent by using the relation

$$
\sigma_{\text {a.c. }}=2 \pi f \varepsilon_{0} \varepsilon^{\prime} \tan \delta
$$

where $f$ is the applied frequency (Tareev 1975).

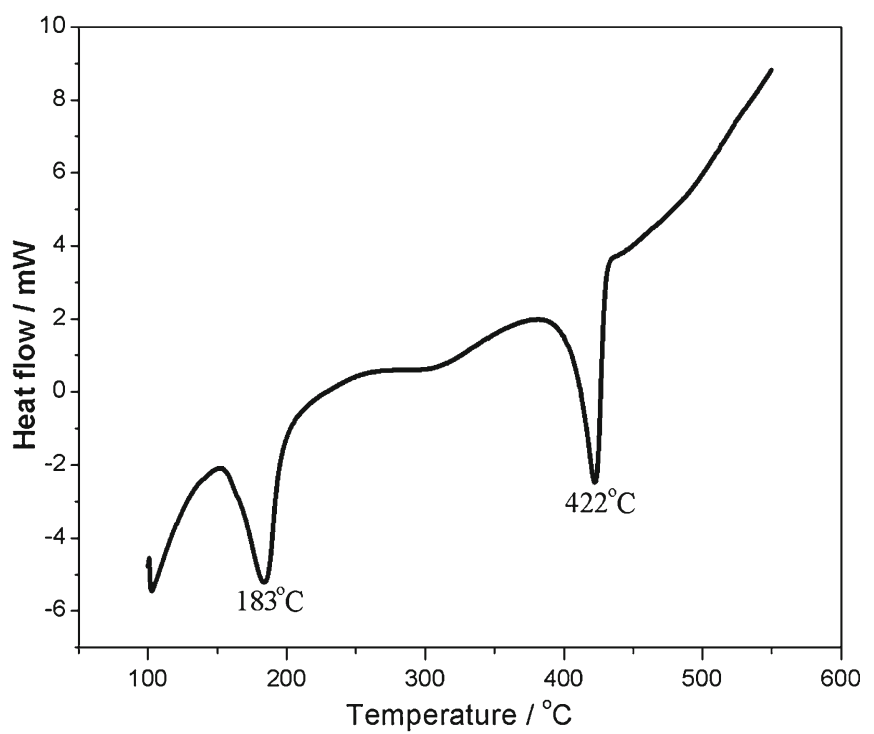

Figure 1. Heat flow diagram obtained by differential scanning calorimetry of gel.

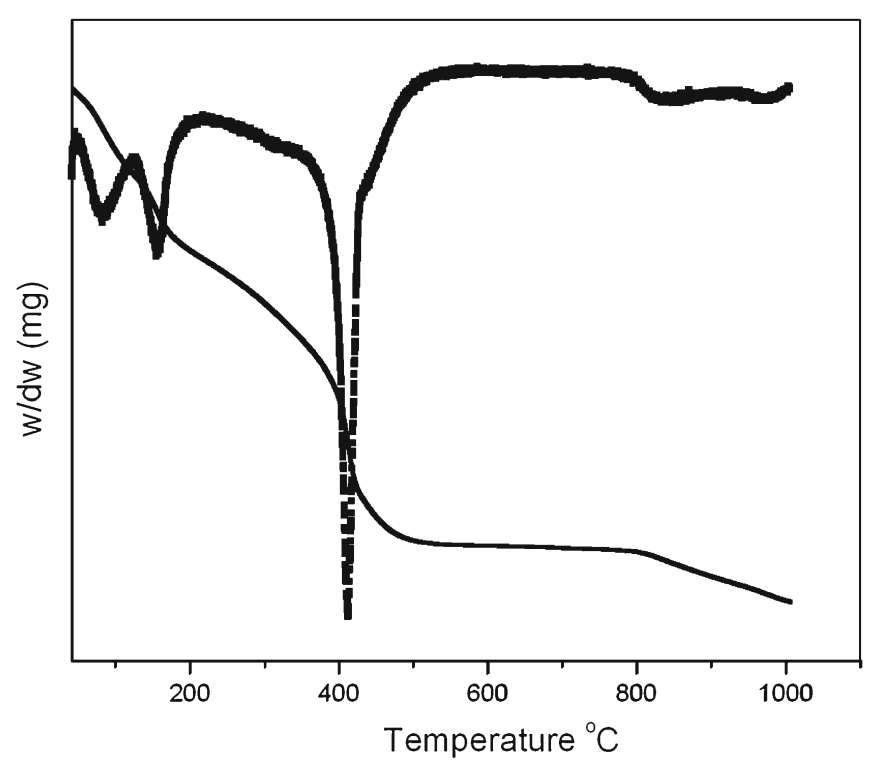

Figure 2. TGA and DTGA curves of gel.

\section{Results and discussion}

\subsection{DSC and TGA}

Figure 1 shows the heat flow diagram of the differential scanning calorimetry performed on the gel. Two exothermic peaks are observed at temperatures $183^{\circ} \mathrm{C}$ and $422^{\circ} \mathrm{C}$. The first exothermic peak at $183^{\circ} \mathrm{C}$ corresponds to the combustion of the gel at this temperature to form the powder. The second peak can be attributed to the formation of the spinel phase at about $400^{\circ} \mathrm{C}$. The calcination trials indicated that the 
phase formation takes place in this temperature range. The crystallites go to a lower energy state by the formation of stable spinel structure through an exothermic stage. In solgel synthesis of $\mathrm{ZnAl}_{2} \mathrm{O}_{4}$ there is an intermediate crystalline phase as indicated in the XRD diagram (figure 3). This intermediate phase has a non-cubical structure and closely resembles a phase of aluminium hydroxide. It appears that this intermediate phase is a mixture of aluminium hydroxide and zinc hydroxide.

Figure 2 depicts TGA of the gel before calcination. Both the variation of mass of the compound with temperature and its rate of variation with temperature (differential coefficient of mass variation with respect to temperature) are shown in

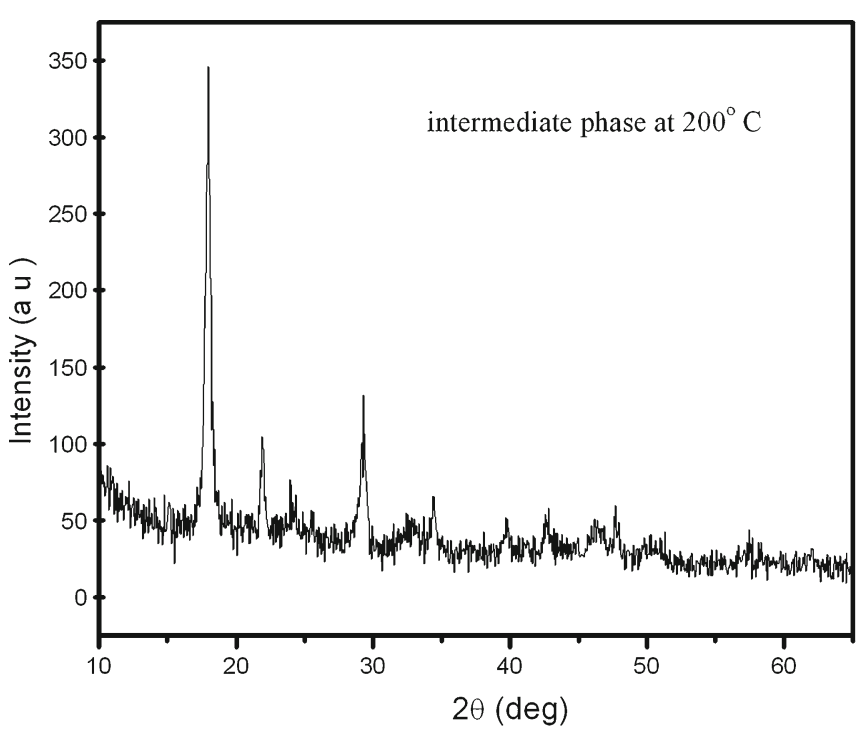

Figure 3. XRD pattern of intermediate phase. the diagram as separate plots. A sudden fall in mass of the compound is evident at two positions, close to $200^{\circ} \mathrm{C}$ and $400^{\circ} \mathrm{C}$. The first one is attributed to the expulsion of the organic solvent in the gel and the formation of the powder hydroxides of zinc and aluminium. The second position can be assigned to the formation of spinel phase structure from the hydroxides of metals. This result is consistent with what was observed in the DSC analysis.

\subsection{Structural studies}

Figure 3 is the XRD diagram of the intermediate phase formed at $180^{\circ} \mathrm{C}$. This compound seems to be a mixture of aluminium hydroxides and zinc hydroxide with a noncubical structure. Figure 4 shows the XRD diagrams of the compound calcinated at $350^{\circ} \mathrm{C}, 500^{\circ} \mathrm{C}, 700^{\circ} \mathrm{C}$ and $800^{\circ} \mathrm{C}$. It can be seen that the phase formation is complete at $700^{\circ} \mathrm{C}$ and further increase in calcination temperature does not improve the crystallinity of the compound. All peaks are well defined pointing to the high crystalline nature of the sample. The XRD pattern was compared with JCPDS files (file no. 74-1138) and all the diffraction peaks are in good match with the reported result and the peaks were indexed with the help of these reported patterns. Rietveld refinement was carried out to verify the formation of spinel phase and also to determine the cation distribution or displacement of zinc ion from A site to B site. It can be observed that excellent matching is obtained in terms of the peak positions and peak intensity between the theoretical and observed data as depicted in figure 5. The lattice parameter obtained from the Rietveld analysis is $8.098 \AA$. The reported value of lattice parameter is $8.099 \AA$ according to the JCPDS data and this close agreement indicates the phase purity of the zinc aluminate

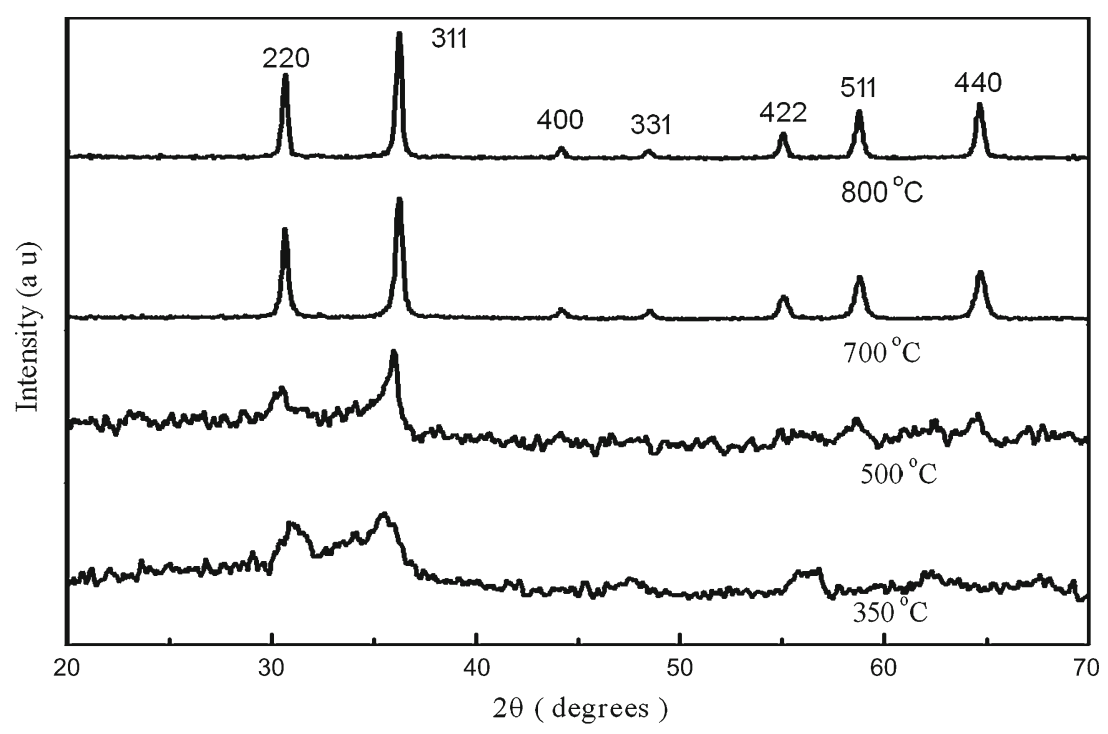

Figure 4. XRD pattern of zinc aluminate particles fired at temperatures starting from $350-800^{\circ} \mathrm{C}$. 


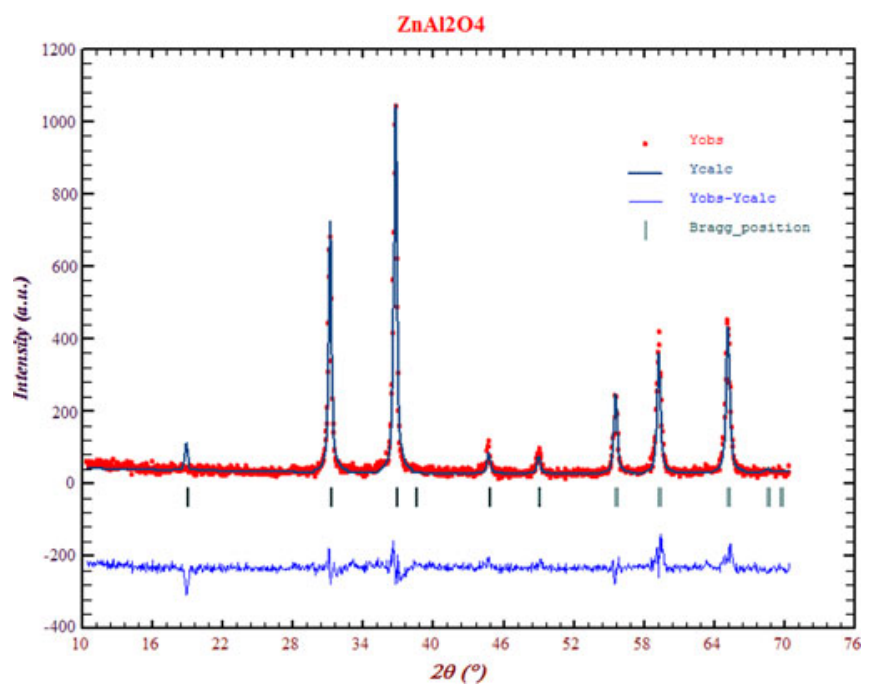

Figure 5. Rietveld analysis of XRD of zinc aluminate particles.

particles obtained in the sol-gel technique. The particle size was determined from the XRD data using the DebyeScherrer equation for the average particle size, $D$

$$
D=\frac{0 \cdot 9 \lambda}{\beta \cos \theta}
$$

where $\lambda$ is the wavelength of the X-ray source, $\beta$ the full width at half maximum (FWHM) of the diffraction peak and $2 \theta$ the diffraction angle (Keer 1998). The FWHM was determined by giving Gaussian fit to the diffraction peaks with the help of a software package. The average particle size was found to be $40 \mathrm{~nm}$.

\subsection{FTIR spectroscopy}

The FTIR spectrum is presented in figure 6: the curves named $\mathrm{A}, \mathrm{B}$ and $\mathrm{C}$ correspond to the gel, the intermediate phase and the final spinel phase of the compounds formed during the sol-gel synthesis. In plot A, a broad absorption band centred at $3470 \mathrm{~cm}^{-1}$ is visible and this corresponds to $\mathrm{OH}$ group, which is contributed by the water content. The bands at $1630 \mathrm{~cm}^{-1}$ and $1386 \mathrm{~cm}^{-1}$ can be attributed to the $\mathrm{OH}$ group in the metal alkoxides present in the gel. An intermediate phase is formed at $180^{\circ} \mathrm{C}$ and the infrared absorption of this material is shown in plot B. It can be seen that the bands at $1630 \mathrm{~cm}^{-1}$ and $1386 \mathrm{~cm}^{-1}$ are still present with a little splitting and this can be due to the formation of metal hydroxides as an intermediate compound before the formation of the zinc aluminate spinel. In plot $\mathrm{C}$, which is of the calcinated zinc aluminate, the band at 3408 is almost absent and a small shallow band is indicated by the slight water content in the crystallites. Three sharp absorption bands visible in plot $\mathrm{C}$ at 682,565 and $510 \mathrm{~cm}^{-1}$ arise from the stretching vibrations of tetrahedral and octahedral bonds in the spinel. The peak observable at $2348 \mathrm{~cm}^{-1}$ can be attributed to the presence of oxygen-oxygen bonds in the $f c c$ crystal lattice

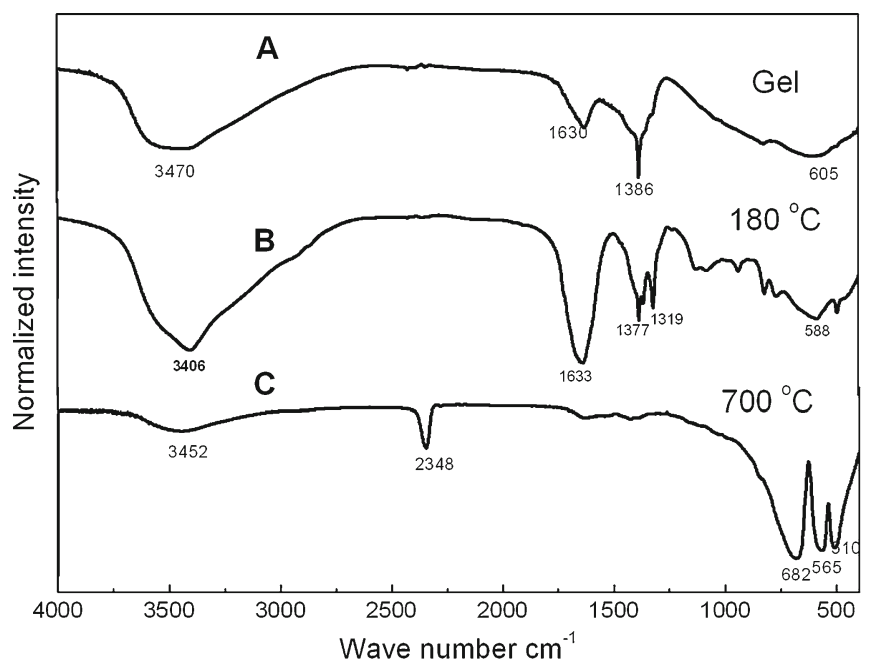

Figure 6. FTIR spectrum of compounds at various stages of synthesis of zinc aluminate nanoparticles.

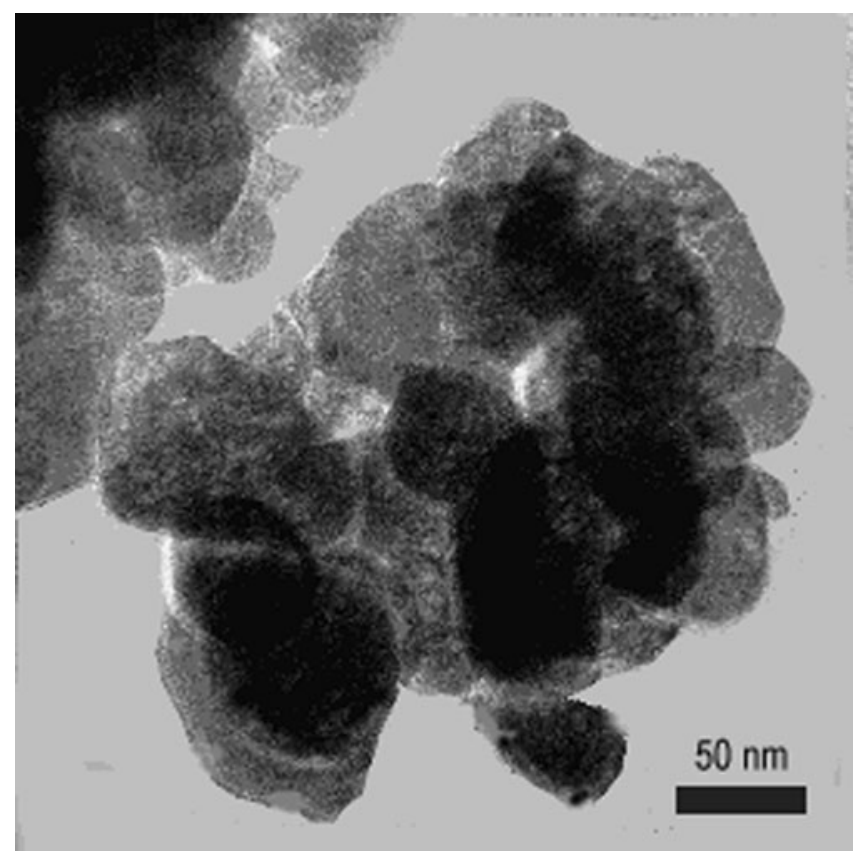

Figure 7. TEM picture of zinc aluminate nanoparticles.

of oxygen atoms. This peak is detectable in all spinel compounds and can be treated as a characteristic feature of spinel structured crystallites. In plot B, the metal bonds start to appear indicating the beginning of the formation of spinel phase.

\subsection{TEM and EDS analysis}

Figure 7 depicts the TEM image of the particles calcinated at $700^{\circ} \mathrm{C}$. The sizes of all particles lie in the range $25-60 \mathrm{~nm}$. The size distribution is narrow. It is evident from the micrograph that the shape of the particles is very nearly spherical. 


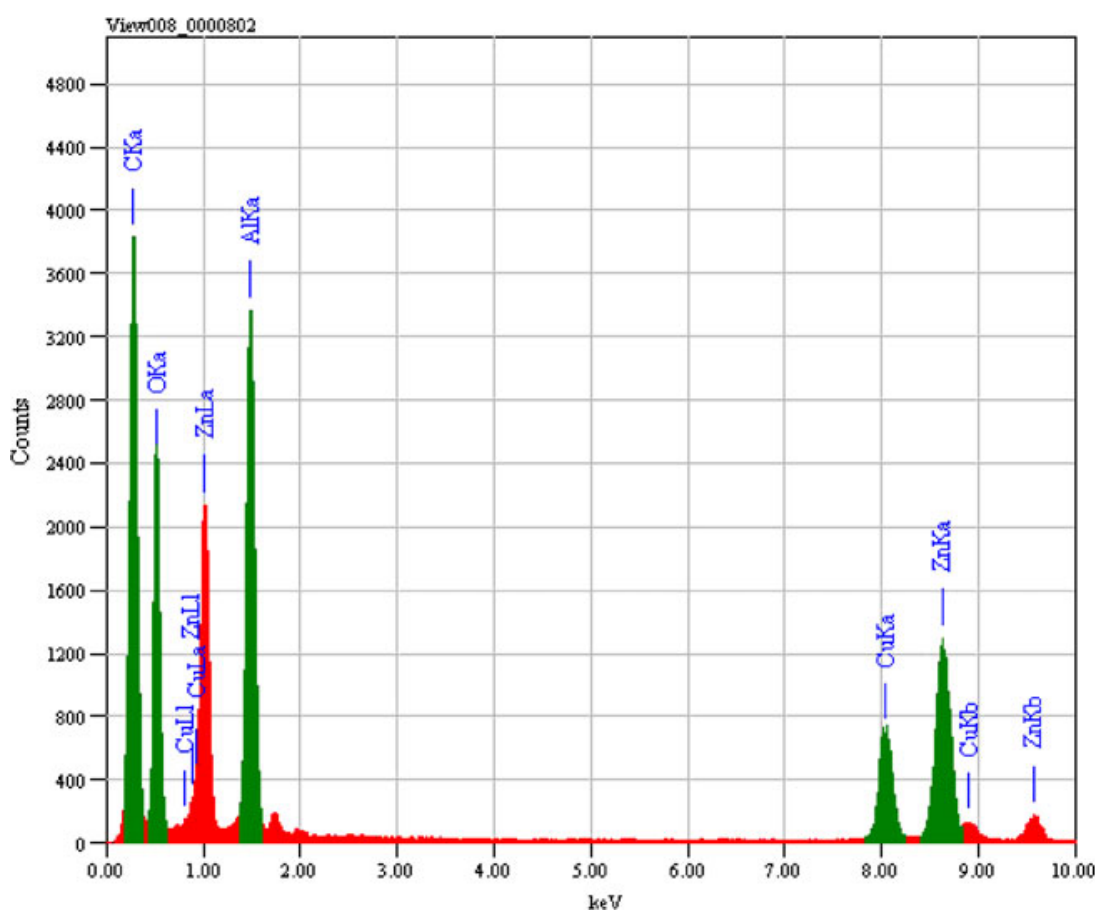

Figure 8. EDS spectrum of zinc aluminate nanoparticles.

The average size of $40 \mathrm{~nm}$ obtained from the XRD analysis is inconsistent with this result.

The energy dispersive spectrum of the particles is depicted in figure 8. The counts corresponding to $\mathrm{C}$ and $\mathrm{Cu}$ are from the organic solvent in which the particles are dispersed and from the copper grid, respectively. The $\mathrm{K} \alpha$ lines of $\mathrm{Al}$ and $\mathrm{Zn}$ can be clearly noticed in the spectrum. An exact quantitative estimate of the content of these elements was not carried out but from the number of counts it appears that the number of atoms of $\mathrm{Zn}$ is half the atoms of $\mathrm{Al}$.

\subsection{Optical measurements}

The absorption spectra of zinc aluminate particles in transmission mode was recorded by dispersing the particles uniformly in liquid paraffin, in the wavelength range of 225$800 \mathrm{~nm}$. For a direct bandgap semiconducting material the absorption coefficient near the band edge is given by

$$
\alpha=\frac{A}{h v}\left(h v-E_{\mathrm{g}}\right)^{1 / 2},
$$

where $\alpha$ is the absorption coefficient, $h v$ the photon energy, $E_{\mathrm{g}}$ the energy gap and $A$ is constant depending on the type of transition. Equation (5), for any energy can be rearranged and written in the form

$$
(\alpha h v)^{2}=A^{2}\left(h v-E_{\mathrm{g}}\right) .
$$

From (6), it is clear when $h v=0, E_{\mathrm{g}}=h v$. The energy gap is determined by plotting $(\alpha h v)^{2}$ against $h v$ and find-

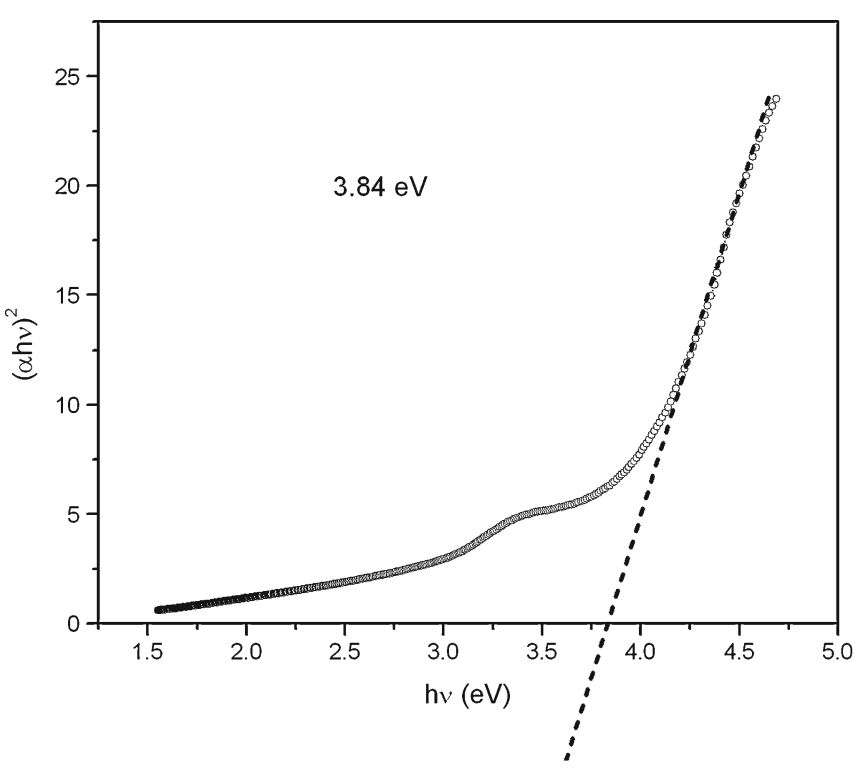

Figure 9. Tauc plot to determine optical bandgap from UV-Vis absorption spectrum.

ing the intercept on the $h v$ axis by extrapolating the plot to $(\alpha h v)^{2}=0$ as shown in figure 9. A bandgap energy of $3.84 \mathrm{eV}$ was observed which is slightly higher than the reported bandgap of bulk zinc aluminate suggesting a blue shift which can be attributed to the quantum confinement due to small size of zinc aluminate particles. 


\subsection{Dielectric studies}

The variation in dielectric permittivity $\left(\varepsilon^{\prime}\right)$ with applied frequency is presented in figure 10 as a semi-log plot in which $\log$ of the frequency is in the x-axis and $\varepsilon^{\prime}$ is in y-axis. It can be observed that there is a steady decrease in $\varepsilon^{\prime}$ with applied frequency. This phenomenon can be understood on the basis of Maxwell-Wagner model of interfacial polarization and Koop's phenomenological theory (Koops 1951; Wagner 1993). The zinc aluminate pellets used in the study was made of pressed nanoparticles. The bandgap studies of the sample already revealed that the sample has semiconducting nature. Every grain of the pellets can be considered having a perfect crystalline characteristics and therefore having low electrical resistance compared to the grain boundaries. This is exactly the model presented in Koop's theory. Maxwell-Wagner type interfacial polarization exists in the grain boundaries around the grains, by accumulation of charges in these high resistance regions. This kind of polarization exists in all polycrystalline condensed matter samples which are heterogeneous in composition (Pohl 1978). Considering that the material has a single relaxation time in the frequency regime under test it is possible to write the equations for complex dielectric permittivity as

$$
\varepsilon^{*}(\omega)-\varepsilon_{\alpha}=\frac{\varepsilon_{\mathrm{s}}-\varepsilon_{\alpha}}{1+i \omega \tau},
$$

where $\varepsilon^{*}(\omega)$ is the complex permittivity at the frequency $\omega$, and $\varepsilon_{\alpha}$ the permittivity at optical frequencies. Equation (7) can be written as real and imaginary parts separated as

$$
\varepsilon^{\prime}(\omega)=\varepsilon_{\alpha}+\frac{\varepsilon_{\mathrm{s}}-\varepsilon_{\alpha}}{1+\omega^{2} \tau^{2}},
$$

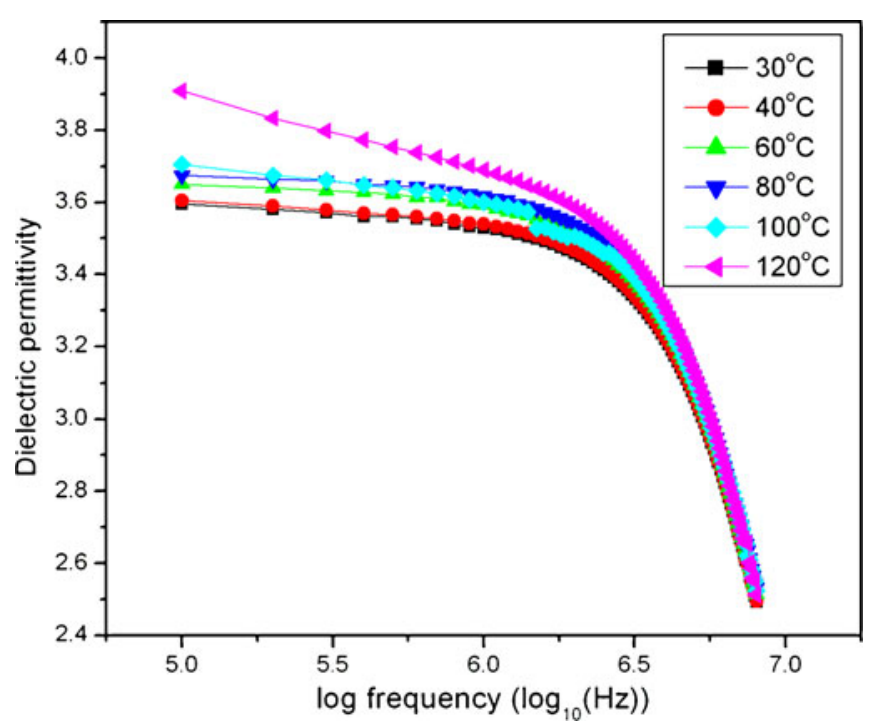

Figure 10. Variation of dielectric permittivity of zinc aluminate with applied frequency at temperatures ranging from $30-120^{\circ} \mathrm{C}$. and

$$
\varepsilon^{\prime \prime}(\omega)=\left(\varepsilon_{\mathrm{s}}-\varepsilon_{\alpha}\right) \frac{\omega \tau}{1+\omega^{2} \tau^{2}} .
$$

Equations (8) and (9) are known as Debye equations since they were derived by Debye on a molecular basis. The decrease in dielectric permittivity with increase in applied frequency is understood according to (8). The presence of interfacial polarization gives rise to a relaxation process with a high relaxation time compared to electronic or dipolar polarization. This causes a decrease in the dielectric permittivity in the given frequencies. It can be observed from figure 10 that there is a steady increase in the permittivity of the material at all frequencies. With the increase of temperature charge carriers acquire more mobility as a result of thermal activation and this can increase the accumulation of charge at the grain boundaries, because the grain boundaries exhibit a lower electrical conductivity. Greater charge accumulation at higher temperatures in the interfaces of grains gives rise to enhanced dielectric permittivity.

It can be observed that the dielectric permittivity of zinc aluminate is smaller than some of the other members in the spinel family. Zinc ferrite and manganese ferrite have larger values of dielectric permittivity (Devan et al 2006; Veena Gopalan et al 2008). Zinc aluminate has a smaller crystal size compared to ferrites. The smaller the size, the smaller is the electronic charge separation in the crystals when an electric field is applied. The component of electronic polarization is smaller in zinc aluminate compared to ferrites. Dielectric loss tangent is plotted against applied frequency in figure 11. The loss tangent decreases with the increase of applied frequency. As the temperature increases, the loss tangent increases steadily and shows shallow relaxation peaks centred at about $2 \mathrm{MHz}$.

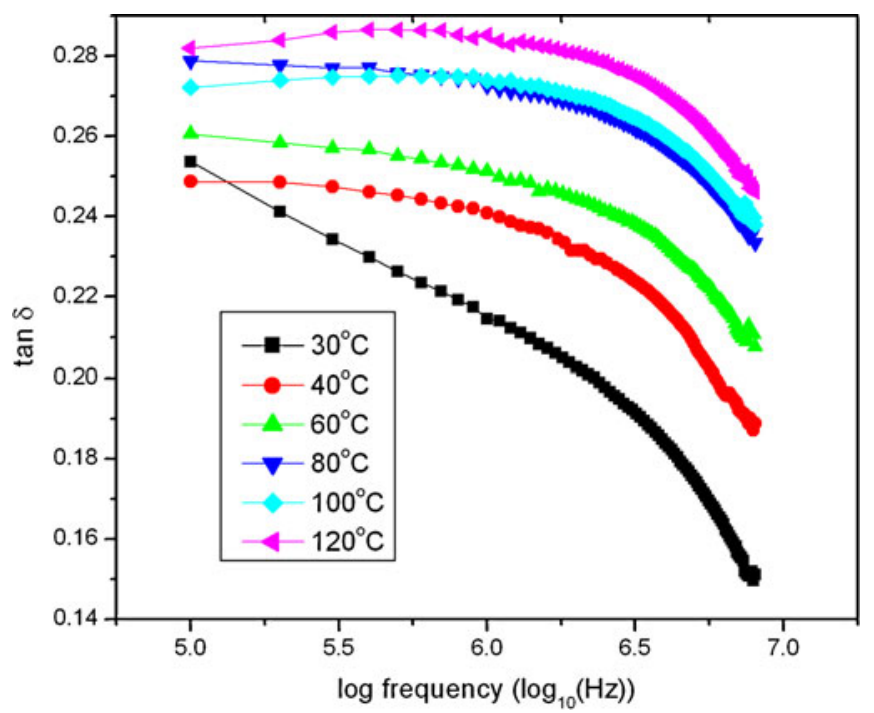

Figure 11. Dielectric loss tangent. 


\subsection{A.c. conductivity studies}

Studies on a.c. conductivity of the zinc aluminate particles were carried out from the loss tangent data by employing the relation given by (2). The variation of a.c. conductivity is plotted in figure 12 as a semi-log plot with frequency in the horizontal axis for temperatures starting from $30-120^{\circ} \mathrm{C}$. At all temperatures a.c. conductivity shows a steady increase with the increase of frequency. In a dielectric material, there are no free charge carriers and, therefore, there cannot be any d.c. conduction by means of the movement of free charges. The a.c. conduction in the material is through a hopping mechanism of bound charges, in which charges hop back and forth between well-defined bound states. Electrons undergo hopping between bound states through a tunneling process from one site to other. The hopping mechanism is supported by the increase in frequency, and a.c. conductivity increases at higher frequencies. Even though an ideal dielectric material does not have any free charge carriers, there can be small amount of free charges in them and the measured conductivity arises from the two contributing factors, viz. the a.c. and d.c. conduction. In general, the measured a.c. conductivity is represented by the relation

$$
\sigma_{\text {a.c. }}(\omega)_{\mathrm{m}}=\sigma_{\text {a.c. }}(\omega)+\sigma_{\text {d.c. }},
$$

where $\sigma_{\text {a.c. }}(\omega)_{\mathrm{m}}$ is the total frequency-dependent conductivity and $\sigma_{\text {a.c. }}(\omega)$ the contribution from the a.c. conductivity and $\sigma_{\text {d.c. }}$ the d.c. conductivity.

The total conductivity and a.c. conductivity are dependent on the angular frequency $\omega$ of the applied signal. From figure 12 , it can be observed that the total conductivity is very small at $100 \mathrm{kHz}$ compared to that at higher frequencies. So the static contribution from the d.c. conductivity is

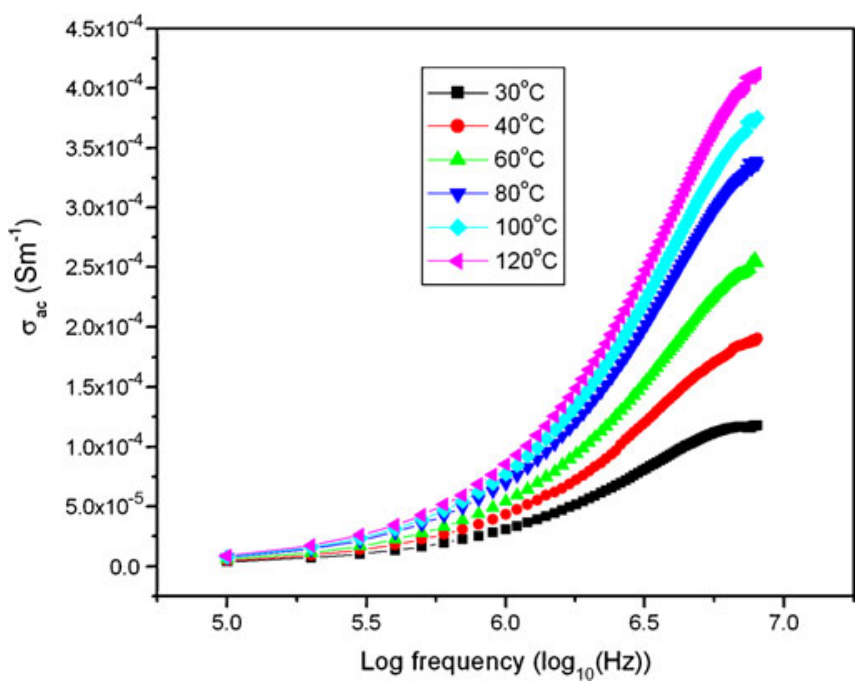

Figure 12. Variation of a.c. conductivity of zinc aluminate with applied frequency at temperatures ranging from $30-120^{\circ} \mathrm{C}$.

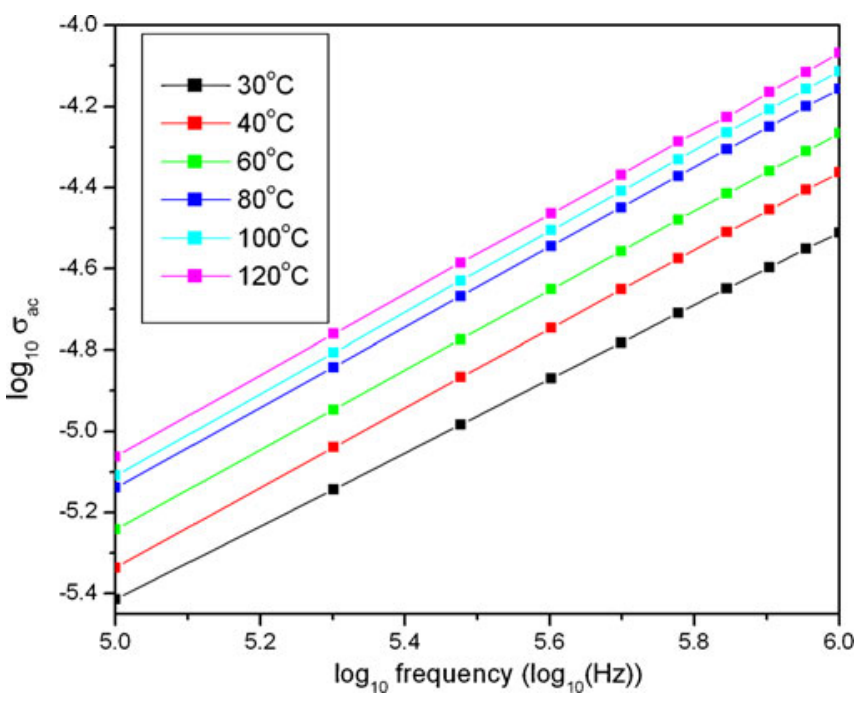

Figure 13. Plots of log of a.c. conductivity against log frequency.

negligible in the total conductivity of the sample at all temperatures. Further, the angular frequency dependence of the a.c. conductivity can be represented in the form

$$
\sigma_{\text {a.c. }}=\sigma_{\text {d.c. }}+A f^{S},
$$

where $A$ and $s$ are coefficients and $f$ the applied frequency (Ghosh et al 2008). Since the d.c. conductivity is found to be negligible, (11) can be written in the form

$$
\sigma_{\text {a.c. }}=A f^{S} \text {. }
$$

The values $A$ and $s$ can be determined by plotting log of a.c. conductivity against $\log$ frequency. The plot is a straight line, since

$$
\log \sigma_{\text {a.c. }}=\log A+s \log f .
$$

Equation (13) is the equation of a straight line with a slope $s$. The values of $s$ for different temperatures were determined by plotting $\log f$ with $\log \sigma_{\text {a.c. }}$ and by finding the slope of the resulting straight line. The plots are presented in figure 13. The plots are parallel to each other and the slope of

Table 1. Frequency exponent factor $s$ and pre-exponent factor $A$ for different temperatures.

\begin{tabular}{lcc}
\hline Temperature $\left({ }^{\circ} \mathrm{C}\right)$ & $s$ & $A\left(\mathrm{~S} \mathrm{~m}^{-1}\right)$ \\
\hline 30 & $0 \cdot 95$ & $9 \cdot 5626 \mathrm{E}-12$ \\
40 & $0 \cdot 95$ & $1 \cdot 4144 \mathrm{E}-11$ \\
60 & 0.95 & $1 \cdot 8019 \mathrm{E}-11$ \\
80 & $0 \cdot 94$ & $2 \cdot 7784 \mathrm{E}-11$ \\
100 & 0.94 & $3 \cdot 0792 \mathrm{E}-11$ \\
120 & 0.94 & $3.4037 \mathrm{E}-11$ \\
\hline
\end{tabular}




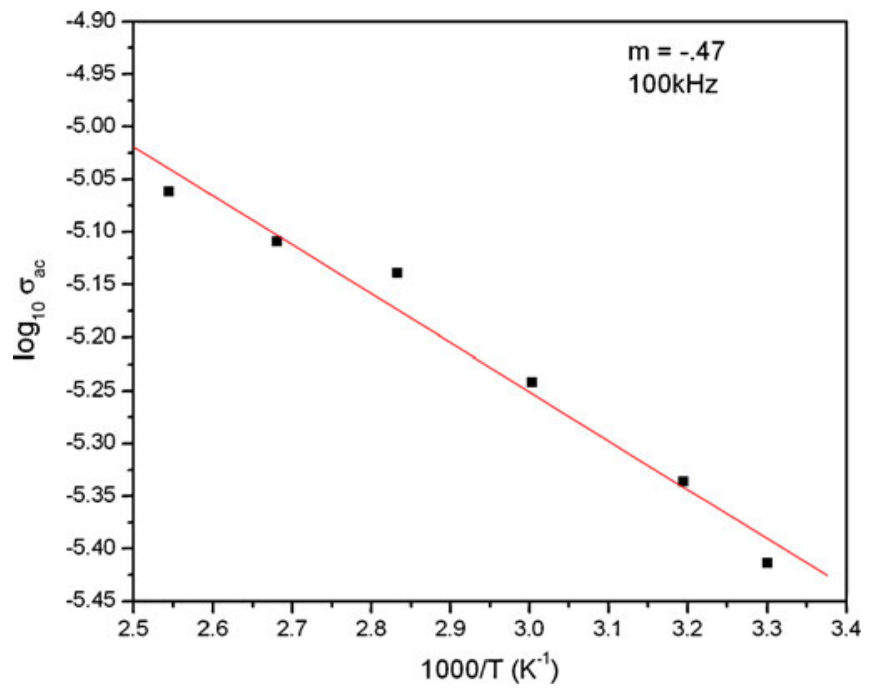

Figure 14. Plot of $\log$ of a.c. conductivity against $1000 / T$ to determine activation energy (for frequency $100 \mathrm{kHz}$ ).

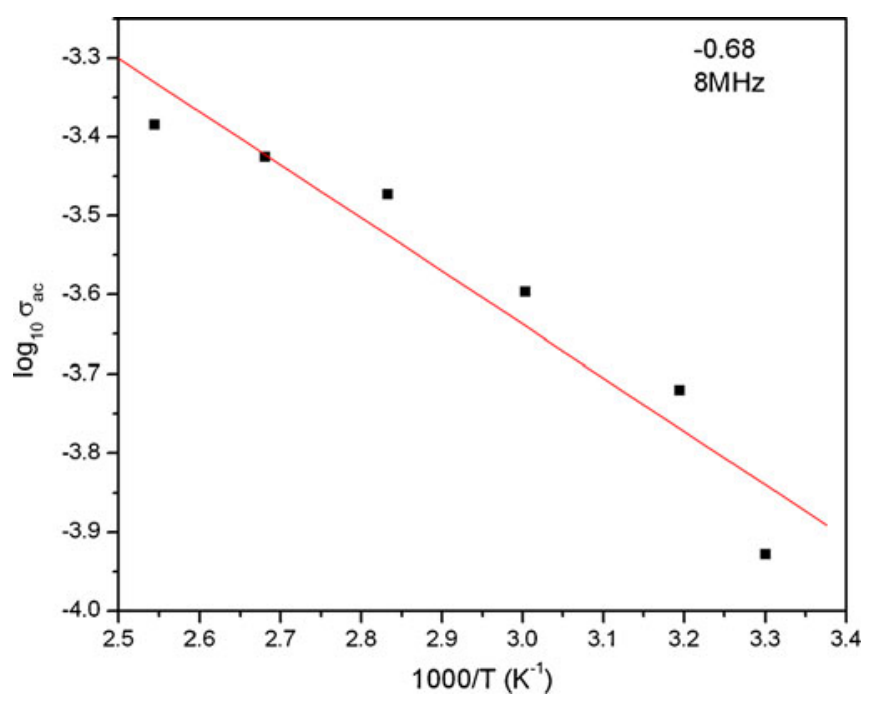

Figure 15. Plot of $\log$ of a.c. conductivity against $1000 / T$ to determine activation energy (for frequency $8 \mathrm{MHz}$ ).

the straight line region is between 0.94 and 0.95 . It is reasonable to assume that the parameter $s$ is independent of temperature. The parameter $s$ can throw light on two distinct conduction mechanisms. The conduction can be through quantum mechanical tunneling (QMT) of charge carriers through barriers separating localized sites and in such a case the parameter $s$ is independent of temperature (El-Samanoudy 2002). Another possible mechanism of conduction is the correlated barrier hopping $(\mathrm{CBH})$ in the same barrier. In $\mathrm{CBH}$ a decrease in the parameter $s$ is expected with increase of temperature. For zinc aluminate particles the decrease in $s$ is only minimal. The values of $A$ were calculated by substituting the value of $s$ in (12). The calculated values of $A$ and $s$ are given in table 1 .
Table 2. Activation energy of a.c. conductivity of zinc aluminate nanoparticles at different frequencies.

\begin{tabular}{lc}
\hline Frequency $(\mathrm{MHz})$ & Activation energy $(\mathrm{eV})$ \\
\hline $0 \cdot 1$ & $0 \cdot 093$ \\
1 & $0 \cdot 110$ \\
2 & $0 \cdot 117$ \\
3 & $0 \cdot 121$ \\
4 & $0 \cdot 123$ \\
5 & $0 \cdot 125$ \\
6 & $0 \cdot 127$ \\
7 & $0 \cdot 131$ \\
8 & $0 \cdot 135$ \\
\hline
\end{tabular}

The a.c. conductivity of zinc aluminate was found to increase with temperature. Absorption of heat energy increases the mobility of electrons taking part in the hopping conduction. The variation of a.c. conductivity with temperature can be understood on the basis of Arrhenius equation given in the form

$$
\sigma_{\text {a.c. }}=\sigma_{0} \exp \left(\frac{-E}{k_{\mathrm{B}} T}\right),
$$

where $\sigma_{0}$ is the pre-exponential factor, $E$ the activation energy, $k_{\mathrm{B}}$ the Boltzmann's constant and $T$ temperature in Kelvin (Elimat et al 2008). Activation energy of zinc aluminate particles was estimated by plotting $\log _{10}\left(\sigma_{\text {a.c. }}\right)$ against $100 / T$ and by finding the slope of the straight line obtained by least square linear fit to the data points. The plots and straight line fits are presented in figures 14 and 15 for frequencies $100 \mathrm{kHz}$ and $8 \mathrm{MHz}$. The activation energy in $\mathrm{eV}$ is given by the equation

$$
E=\frac{2 \cdot 303 \times 1000 \times m \cdot k_{\mathrm{B}}}{e}
$$

where $m$ is the slope and $e$ the electronic charge. The activation energy was found to increase with frequency and the values obtained for different frequencies are presented in table 2. Activation energy in the range of $0 \cdot 1 \mathrm{eV}$ suggests that the a.c. conduction in the material is realized through electrons. QMT of electrons between barriers can be the possible mechanism of a.c. conduction in zinc aluminate particles because there is no appreciable change in the parameter $s$ with temperature.

\section{Conclusions}

The formation of spinel structured zinc aluminate nanoparticle with average size of $40 \mathrm{~nm}$ was accomplished through a sol-gel combustion process. It was found that an intermediate phase possibly of a mixture of aluminium hydroxide and zinc oxide is formed at $180^{\circ} \mathrm{C}$. The spinel phase was formed at a temperature close to $400^{\circ} \mathrm{C}$ and this was established by calcination trials from $350-800^{\circ} \mathrm{C}$. The optical 
bandgap of the material is found to be $3.84 \mathrm{eV}$, slightly greater than that of bulk material and this is attributed to the quantum confinement due to small size of zinc aluminate particles. The dielectric permittivity of the material exhibits dispersion in the low-frequency region and interfacial polarization of Maxwell-Wagner type could be the reason for this phenomenon. A.c. conductivity increases with frequency. The frequency exponent factor was found to be nearly unity and it did not show any appreciable variation with temperature. This is attributed to a.c. conduction through hopping of electron between sites by means of quantum mechanical tunneling. The zinc aluminate is a normal spinel and no evidence for cation redistribution is found from Rietveld analysis. Therefore, it is to be assumed that the non-magnetic counterpart of zinc ferrite in the nano-regime is not susceptible to size effects and the cation distribution does not undergo any change as evident from XRD and Rietveld analysis. However, high surface area zinc aluminate can be synthesized and optical studies indicate that they can be suitable for other optical applications. The size effect on the optical properties can be an interesting piece of investigation for further studies.

\section{Acknowledgement}

One of the authors (EMAJ) acknowledges the fellowship granted by UGC, India, under the Faculty Improvement Programme.

\section{References}

Ciupina V, Carazeanua I and Prodan G 2004 J. Optoelectron. Adv. M. 61317

Devan R S, Kolekar Y D and Chougule B K 2006 J. Phys.: Condens. Matter 189809

Elimat Z M, Zihlif A M and Ragosta G 2008 J. Phys. D: Appl. Phys. 41165408

El-Samanoudy M M 2002 J. Phys.: Condens. Matter 141199 PII: S0953-8984 (02) 26167-6

Ghosh A, Bhattacharya S, Bhattacharya D P and Ghosh A 2008 J. Phys.: Condens. Matter 20035203

Keer H V 1998 Principles of solid state physics (New Delhi: Wiley Eastern Ltd.)

Koops C G 1951 Phys. Rev. 831

Mohammed E M and Anantharaman M R 2002 J. Instrum. Soc. India 32165

Pandey R, Gale J D, Sampath S K and Recio J M 1999 J. Am. Ceram. Soc. 823337

Pohl H A 1978 Dielectrophoresis (London: Cambridge University Press)

Roy M K, Haldar B and Verma H C 2006 Nanotechnology 17232

Tareev B 1975 Physics of dielectric materials (Moscow: Mir Publishers)

van der Laag N J, Snela M D, Magusin P C M M and de With G 2004 J. Eur. Ceram. Soc. 242417

Veena Gopalan E, Malini K A, Saravanan S, Sakthi Kumar D, Yoshida Y and Anantharaman M R 2008 J. Phys. D: Appl. Phys. 41185005

Wagner K W 1993 Ann. Phys. 40817

Wei X and Chen D 2006 Mater. Lett. 60823 